\title{
From the Co Nanocrystals to Their Self-Organizations: Towards Ferromagnetism at Room Temperature
}

\author{
I. LISIECKI* \\ Laboratoire LM2N, UMR CNRS 7070, Université P. et M. Curie \\ Bât F, 4 Pl. Jussieu, 75005 Paris, France
}

\begin{abstract}
Here, we show that rather uniform $7 \mathrm{~nm}$ Co nanocrystals self-organize into long-range two- and three-dimensional superlattices. Due to this ability we have to control the mesoscopic ordering; unexpected intrinsic collective chemical and physical properties have been discovered. By annealing treatment, a crystallographic transition from a polycrystalline fcc- to single crystalline hcp-Co phase is obtained leading to a drastic change in the magnetic properties such as an increase in the blocking temperature that can reach a value close to room temperature. Neither coalescence between nanocrystals nor oxidation of the Co material is observed. In these artificial solids, when the individual nanocrystal anisotropy is low, super-spin glass behavior is observed.
\end{abstract}

PACS: 75.50.Tt, 75.70.Cn

\section{Introduction}

The organization of identical passivated nanometer-scale particles into micrometer-scale ordered arrays constitutes a new generation of materials. They cover a large variety of structures including chains (1D), compact hexagonal arrays (2D), 3D superlattices called supracrystals, rings and honey-like structures $[1,2]$. Several strategies for self-assembling the nanocrystals exist: (i) The self-assembly in solution is governed by the balance of attractive and repulsive forces between nanocrystals [3]. (ii) The deposition on a substrate layer by layer is related to liquid-gas phase transition [4]. (iii) The shape of the template predefined the nanocrystal arrangements when strong interactions between the nanocrystals and templates take place [5-7]. (iv) Self-assembly at the liquidliquid, liquid-air and liquid-solid interface takes place [8].

As a result of the periodic ordering of the nanocrystals, this new generation of materials from metallic, semiconductor and magnetic nanocrystals exhibit unique mechanical [9], transport [10], magnetic [11-14], optical [15-17], and vibrational [18-20] intrinsic physical properties. For example, it is well known that color in precious opal results from the high ordering of the uniform silica spheres [21]. Intrinsic chemical properties related to their stability against oxidation $[22,23]$ and robustness $[24,25]$ due the nanocrystal ordering has been recently discovered. All these changes in the chemical and physical properties give rise to an enormous interest for exploiting these collective properties and the possibility of using

* e-mail: isabelle.lisiecki@upmc.fr them in functional devices. Magnetic nanocrystals have attracted much interest in recent years as they have many potential technological applications, the most significant being in high-density data storage [26-28]. Here, we concentrate on the self-organisations of cobalt nanocrystals, their preparation and the chemical and physical properties resulting from their 2D and 3D mesoscopic ordering. We also propose an annealing strategy for converting the particle structure from Co polycrystal to Co-hcp single-crystalline phase, highly required due to its high anisotropy.

\section{2D and 3D superlattices of Co nanocrystals.} Characterisation and chemical properties

\subsection{Synthesis of dodecanoic acid coated Co nanocrystals}

The synthesis of Co nanocrystals [29] takes place in pure micelles of cobalt(II) bis(2-ethylhexyl) sulfosuccinate, $\mathrm{Co}(\mathrm{AOT})_{2}[30]$, the size and form of which are controlled by the water content, defined as $w=\left[\mathrm{H}_{2} \mathrm{O}\right] /$ $[\mathrm{AOT}]=32$. The cobalt ions are reduced by addition of sodium borohydride, $\mathrm{NaBH}_{4}$, to the micellar solution. The concentration of reducing agent is defined as $R=$ $\left[\mathrm{NaBH}_{4}\right] /\left[\mathrm{Co}(\mathrm{AOT})_{2}\right]$. After synthesis, the Co nanocrystals are extracted from the AOT surfactant by adding dodecanoic acid molecules that covalently bond [31] to the metallic surface. After being washed with alcohol, the particles are dispersed in hexane. At $R=0.5$, the micellar solution remains isotropic and the nucleation and growth processes give rise to a single population of nanocrystals. On increasing the $R$ value above 1 , the micelles are destroyed because of the limiting water concentration and two nanocrystal populations are produced. 
In that case, the solution is centrifuged to precipitate bulk cobalt and larger particles, and we recover only the smaller nanocrystals.

TABLE

Average diameter of Co nanocrystals, $D$, and size distributions $\sigma$, at various $R$ values.

\begin{tabular}{c|c|c|c|c|c|c}
\hline \hline$R$ & 0.5 & 1 & 2 & 4 & 6 & 8 \\
$D[\mathrm{~nm}]$ & 6 & 7 & 7 & 7 & 7 & 8 \\
$\sigma[\%]$ & 30 & 18 & 13 & 12 & 12 & 8
\end{tabular}

The increase in the reducing agent concentration from $R=0.5$ to $R=8$ induces an increase in the mean size of the nanocrystals from $6 \mathrm{~nm}$ to $8 \mathrm{~nm}$ (Table). At the same time, the size distribution is found to significantly decrease from $29 \%$ to $8 \%$ (Table). This behavior has mostly to be related to the excess of reducing agent that modifies the nucleation of growth processes. Reduction of cobalt ions is not complete below $R$ equal to 8 [32]. Therefore, the smaller nanocrystals obtained at low $R$ values (and that can be considered as seeds) grow with increasing $R$ value, i.e., with increasing the yield of the reduction reaction. Besides, during the size selection that occurred at the end of the synthesis, only the smaller nanocrystals are collected. Hence, the amount of reducing agent is one of the key parameters in controlling the size distribution of cobalt nanocrystals.

\subsection{From Co nanocrystals to the 2D and $3 D$ superlattices}

Due to Van der Waals interactions and when the size distribution is equal or lower than $13 \%$ [29], Co nanocrystals 2D and 3D self-organize. Transmission electron microscopy (TEM) image (Fig. 1A) shows that the dropwise deposition of the colloidal solution on a TEM grid covered with a thin layer of highly ordered pyrolytic graphite (HOPG) spontaneously leads to the organization of nanocrystals in $2 \mathrm{D}$ hexagonal network over a long range. The mean diameter and size distribution of the nanocrystals are $7.2 \mathrm{~nm}$ and $11 \%$, respectively, and the interparticle gap is $2 \mathrm{~nm}$. In regard to the length of the dodecanoic acid molecule in the all-trans conformation, $1.77 \mathrm{~nm}$ [33], the found interparticle gap thus points to a dense lateral packing. By using the same deposition process with an increased solution concentration, Co nanocrystals 3D self-organize (Fig. 1B). The steps show the formation of 3D assemblies from one layer (a) to a multilayer system $((\mathrm{b})-(\mathrm{d}))$, thin enough to be observed by TEM. Thicker 3D superlattices or supracrystals are prepared by slowly evaporating a highly concentrated colloidal solution on HOPG at a substrate temperature maintained at $25^{\circ} \mathrm{C}$ by a Peltier module [34]. The scanning electron microscopy (SEM) image presented in Fig. 1C shows that the film of supracrystals appears cracked. Its thickness varies from a few micrometers on the border to tens of nanometers in the central part.
The corresponding small-angle X-ray diffraction pattern (GISAXRD) (Fig. 1D) shows several reflections characteristic of long-range order of the nanocrystals in a face-centered cubic (fcc) suprastructure growing in the [111] direction [34]. The (111) reflection is very narrow and nearly resolution-limited $\left(0.0045 \mathrm{~nm}^{-1}\right)$, indicating long-range out of plane order [34]. This gives us the minimum value for the coherence length, $140 \mathrm{~nm}$. From the (111) reflection, we deduce a stacking periodicity equal to $11 \mathrm{~nm}$ including the metal core and a coating contribution and gives us an interparticle gap of $3 \mathrm{~nm}$.

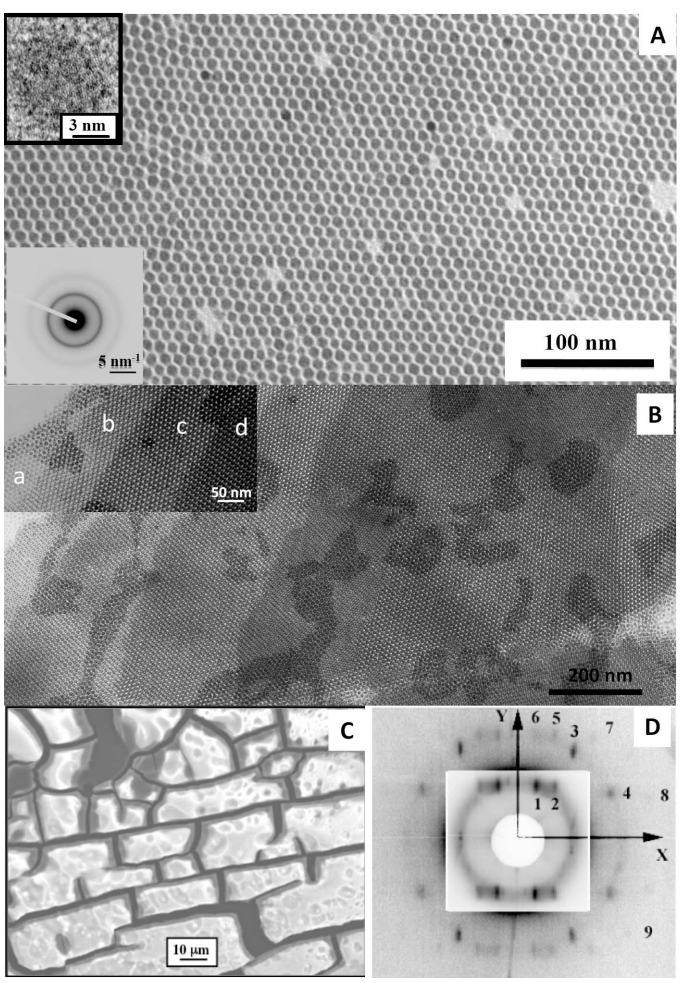

Fig. 1. (A) TEM image of $7.2 \mathrm{~nm}$ cobalt nanocrystals ordered in a compact hexagonal monolayer. Top inset: high-resolution pattern of a single cobalt nanocrystal. Bottom inset: electron diffraction pattern. (B) TEM image of thin 3D organizations. (C) SEM image of supracrystals. (D) Corresponding X-ray diffraction pattern.

Due to the interdigitation of the alkyl chains, these long-range 2D and 3D superlattices are highly stable over several weeks, and neither oxidation of the material nor coalescence between the nanocrystals is observed [14].

\subsection{Control of the Co crystallinity}

The organization of identical passivated nanometer-scale magnetic particles into micrometer-scale ordered arrays will facilitate their use in regard to applications such as ultrahigh density recording [27, 28]. However, with decreasing particle size, we come into conflict with the superparamagnetism caused by the reduction of the anisotropy energy per particle [35-39]. To overcome this 
problem, the use of hard magnetic nanomaterials is required. Due to its high magnetic moment per nanocrystal (higher than FePt), hcp-Co is a good candidate.

The as-synthesized Co nanocrystals obtained in reverse micelles are polycrystalline. The electron diffraction pattern is characterized by two diffuse rings related to lattice spacings of $2.04 \AA( \pm 0.04 \AA)$ and $1.20 \AA$, which could be indexed as the (111) and (220) reflections of fcc Co respectively (bottom inset, Fig. 1A). High-resolution TEM (HRTEM) shows that these nanocrystals are composed of a few ordered domains, less than $1 \mathrm{~nm}$ in size (top inset, Fig. 1A). One strategy to obtain the magnetically hard hcp phase is to recrystallize the magnetically soft cubic-Co nanocrystals through a post-synthesis thermal treatment. Such a treatment is not trivial as in many systems (e.g., FePt, CoPt and Co) [40-44] it leads to an undesirable nanocrystal coalescence and/or oxidation. Hence, after deposition, the 2D and 3D superlattices are placed in a closed quartz ampule with a nitrogen atmosphere and are annealed in a furnace at various temperatures $\left(250,300\right.$ and $350{ }^{\circ} \mathrm{C}$ ) for $15 \mathrm{~min}$. At $250^{\circ} \mathrm{C}$, the nanocrystal structure significantly changes.

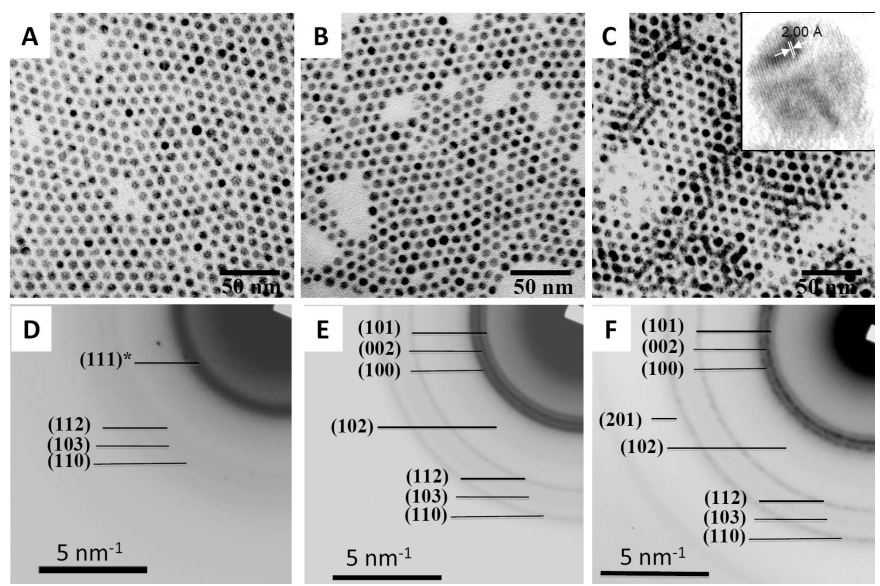

Fig. 2. TEM images of Co nanocrystals ordered in a compact hexagonal monolayer in situ annealed at (A) $250{ }^{\circ} \mathrm{C}$, (B) $300{ }^{\circ} \mathrm{C}$, (C) $350^{\circ} \mathrm{C}$. Inset: high-resolution pattern of a hcp-Co single-crystal. (D), (E) and (F) corresponding electron diffraction patterns.

The diffraction pattern made on a $2 \mathrm{D}$ superlattice (Fig. 2D), shows in coexistence with the diffuse ring related to the likely (111) reflection of fcc Co, three discrete (also still somewhat diffuse) rings (the external triplet) characterized by $1.29 \pm 0.04,1.20 \pm 0.04$ and $1.10 \pm 0.04 \AA$ distances corresponding respectively to (110), (103) and (112) planes of the hcp-Co phase. After annealing at $300^{\circ} \mathrm{C}$, the (111) ring splits into three discrete rings (the internal triplet) characterized by $2.23 \pm 0.04,2.1 \pm 0.04$ and $1.99 \pm 0.04 \AA$ corresponding respectively to (100), (002) and (101) of the hcp-Co phase.

In addition, to the hcp internal and external triplets, the diffraction pattern shows one ring characterized by distance of $1.47 \pm 0.04 \AA$, corresponding to the hcp (102) spacing's (Fig. 2E).

At $350{ }^{\circ} \mathrm{C}$, we observe, similarly to the $300^{\circ} \mathrm{C}$ annealed sample, all the reflections corresponding to a pure hcp structure, with an additional one characterized by a distance $1.03 \pm 0.04 \AA$, corresponding to the hcp (201) spacing (Fig. 2F). This behavior indicates the progressive disappearance of the likely fcc-Co phase in favor of the hcp-Co phase. At $350^{\circ} \mathrm{C}$, the HRTEM images (inset, Fig. 2C) mostly show hcp-Co single crystals. At this temperature, neither coalescence (Fig. 2C) nor detectable oxidation is observed. At all annealing temperatures investigated, Co nanocrystals remain long-range $2 \mathrm{D}$ self-organized in a hexagonal network (Fig. 2A-C) [35].

The $3 \mathrm{D}$ fcc supracrystals, submitted to the same thermal treatment, remain also highly stable [35, 45]. Hence, the suprastructure is not only maintained but it is also improved during annealing as evidences by small angle $\mathrm{X}$-ray scattering (SAXS) study indicating an increase in the coherence length in the supracrystals. This behavior is accompanied by a systematic decrease in the interparticle distance. These structural changes are enabled by the coating chain melting, which allows a significant re-arrangement of the nanocrystals. The high thermal stability of these artificial solids is attributed to the robustness of dodecanoic acid molecules surrounding the nanocrystals.
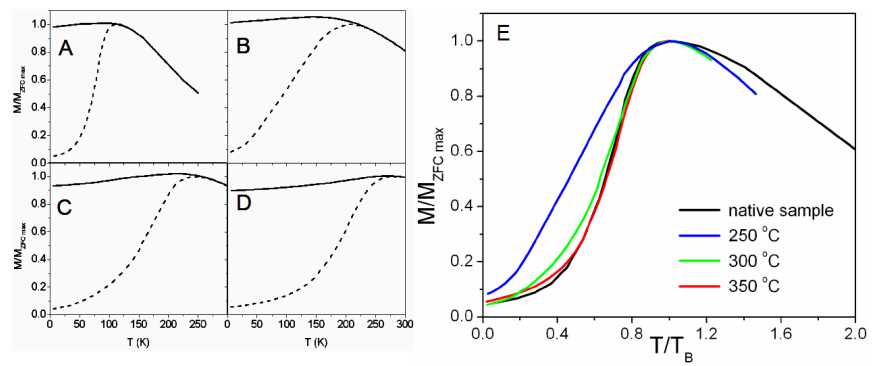

Fig. 3. FC (full lines), and ZFC (dashed lines) $M$ vs. $T$ curves of supracrystals (A) native and annealed at $(\mathrm{B})$ $250{ }^{\circ} \mathrm{C}$, (C) $300^{\circ} \mathrm{C}$, (D) $350^{\circ} \mathrm{C}$. (E) ZFC $M$ vs. $T / T_{\mathrm{B}}$ curves for the supracrystals.

Figure 3 shows the field cooled (FC) and zero field cooled (ZFC) magnetization versus temperature curves, measured with an applied field of 20 Oe, for fcc supracrystals in the native state (A) and annealed at 250, 300 and $350^{\circ} \mathrm{C}$ (Fig. 3B-D, respectively). As shown, the thermal treatment of supracrystals induces a progressive increase in the blocking temperature $T_{\mathrm{B}}$ from $\approx 100 \mathrm{~K}$ for the native state to $\approx 280 \mathrm{~K}$ when $T_{\text {ann }}=350{ }^{\circ} \mathrm{C}$. This behavior is explained by the crystallographic transition from a polycrystalline and probably fcc-cobalt phase to the single crystalline hcp-Co phase, i.e. by an increase in the magnetic anisotropy.

After the annealing at $250^{\circ} \mathrm{C}$, we observe that the normalized ZFC peak to the $T_{\mathrm{B}}$ is broadened with respect to the native sample (Fig. $3 \mathrm{E}$ ). At this stage, the crystallographic transition is not complete as revealed by the 
electron diffraction study, leading to a distribution of the anisotropy in the sample and then to a distribution of the barrier energies. After annealing at higher temperature, 300 and $350^{\circ} \mathrm{C}$, a progressive narrowing of the $\mathrm{ZFC}_{\text {norm }}$ peak is observed until the native width is recovered. This behavior is attributed to the crystallographic transition that is complete at $350^{\circ} \mathrm{C}$.

This results shows that annealing of Co nanocrystals convert the nanomaterial from soft to hard magnetic nanomaterial. This treatment does not result in either oxidation or coalescence of the nanocrystals self-organized in $2 \mathrm{D}$ and $3 \mathrm{D}$ superlattices. At $350^{\circ} \mathrm{C}$, the fcc supracrystals are ferromagnetic near room-temperature value.

\subsection{Control of the mesoscopic ordering of $3 D$ superlattices}

By controlling the substrate temperature during the solvent evaporation process, mesoscopic ordering of $3 \mathrm{D}$ assemblies of Co nanocrystals can be controlled [46].

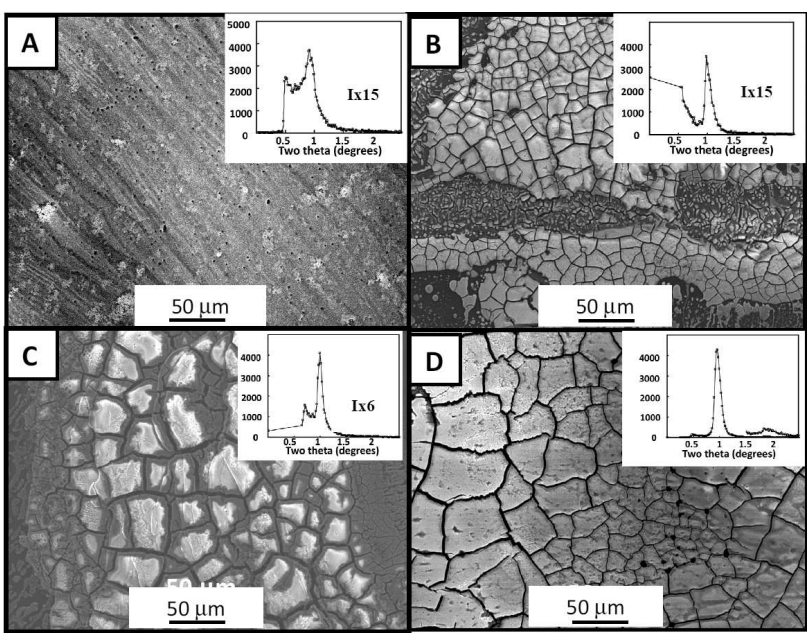

Fig. 4. SEM images of supracrystals obtained at various substrate temperatures (A) $T=10^{\circ} \mathrm{C}$, (B) $T=$ $25^{\circ} \mathrm{C}$, (C) $T=35^{\circ} \mathrm{C}$, (D) $T=45^{\circ} \mathrm{C}$. Insets: corresponding diffractograms.

The substrate temperature is controlled by a Peltier cooling stage. Nitrogen is used to prevent cobalt oxidation. Its flow can be adjusted; the evaporation rate is presently characterized by the hexane partial pressure, kept at $c a .40 \%$ of the saturation value in the evaporation cell. The cobalt nanocrystals are characterized by an average size and size distribution of $7.2 \mathrm{~nm}$ and $12 \%$, respectively.

In the temperature range $5<T<12{ }^{\circ} \mathrm{C}$, the deposition gives rise to the formation of a thin film morphology (less than $500 \mathrm{~nm}$ ) with a smooth surface (Fig. 4A). The diffraction pattern reveals a diffuse ring indicating the absence of any long-range ordering. This is further confirmed by the diffractogram showing a low-intensity peak characterized by a broad half-width at half-maximum (inset, Fig. 4A). By increasing the temperature, $18<T<25^{\circ} \mathrm{C}$, the morphology of the film drastically changes.

Most of the nanomaterial is concentrated at the center of the substrate where the films appear cracked to give blocks of random size and shape (Fig. 4B-D). Upon increasing the temperature, the blocks become increasingly widespread. The SAXS study indicates a long-range fcc structure $[34,46]$. At $25^{\circ} \mathrm{C}$, the diffractogram shows that the (111) reflection width is nearly resolution-limited. By increasing the temperature from 25 to 35 and $45^{\circ} \mathrm{C}$, the peak width decreases and becomes increasingly intense (inset, Fig. $4 \mathrm{~B}-\mathrm{D}$ ). At $45^{\circ} \mathrm{C}$, a second-order reflection appears (inset, Fig. 4D). This behavior clearly indicates an increase in both size and coherence length of supracrystals.

These results clearly show that the temperature is a key parameter to control the mesoscopic ordering of 3D assemblies of Co nanocrystals, i.e. to control the nanocrystal diffusion within the solution and on the substrate. Hence, with the same batch of nanocrystals, we can produce either long-range fcc supracrystals or disordered assemblies.

\subsection{Enhanced stability of Co hcp single crystals induced by the mesoscopic ordering}

To probe the stability against oxidation of hcp-Co single crystals, they are exposed to air for some hours $[22,23]$.
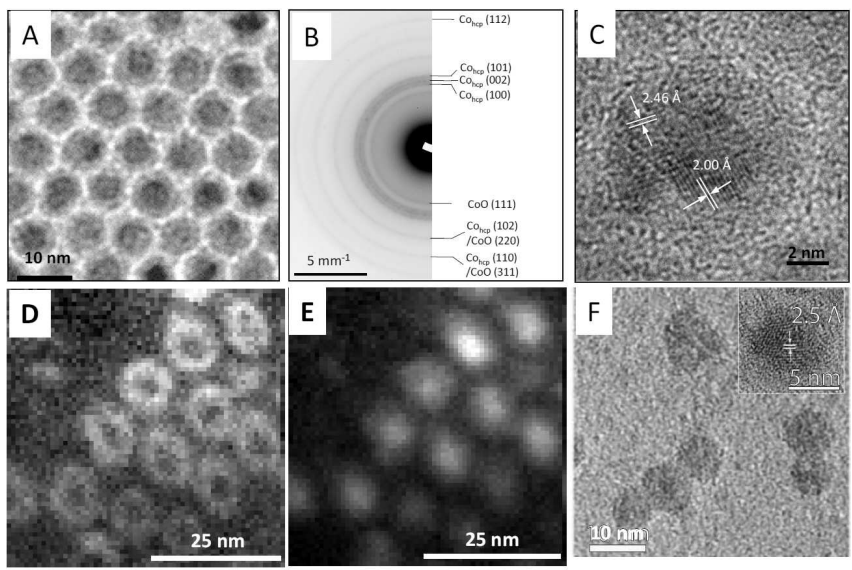

Fig. 5. (A) TEM image of core/shell $\mathrm{Co}_{\mathrm{hcp}} / \mathrm{CoO}$ nanocrystals ordered in a compact hexagonal monolayer. (B) Corresponding electron diffraction pattern. (C) HRTEM image of one of the nanocrystals in (A).

(D) Elemental map for oxygen, using the $\mathrm{OK}$ edge;

(F) elemental map for cobalt, using the Co $L$ edge.

(F) TEM image of $\mathrm{CoO}$ nanocrystals. Inset: corresponding HRTEM image.

2D superlattices of hcp-Co single crystals are prepared by using the in situ annealing process at $350^{\circ} \mathrm{C}$ described in Sect. 2.3. The Co nanocrystals are characterized by a mean size and size distribution of $7.5 \pm 0.4 \mathrm{~nm}$ and $9.5 \%$, 
respectively. After exposure to air for few hours, TEM image (Fig. 5A) shows that nanocrystals do not present anymore a homogeneous aspect (Fig. 2C) but are now characterized by a core-shell contrast.

In addition to the hcp-Co signature, the electron diffraction pattern (Fig. 5B) displays a ring at $2.46 \AA$ indicative of the (111) lattice spacing of the cubic CoO. The HRTEM image showing a single nanocrystal in a hexagonal array (Fig. 5C) reveals a core with lattice planes characterized by a distance of $2.00 \AA$, attributed to the (002) planes of hcp-Co. The shell, which appears poorly crystallized, reveals some lattice planes with a distance of $2.46 \AA$ typical of the (111) planes of CoO. These results clearly indicate the formation of core/shell nanocrystals composed of highly crystallized hcp-Co as the core and poorly crystallized $\mathrm{CoO}$ as the shell.

Electron energy loss spectroscopy (EELS) study performed on this sample further confirms this claim. The elemental map for oxygen, using the O $K$-edge (Fig. 5D) shows that the brightness is mainly located in the shell region while it is clearly observed in the core region when the elemental map is obtained for Co, using Co $L$ edge (Fig. 5E) [22]. So, when Co nanocrystals, closed-packed in a 2D hexagonal array, are submitted to air, they transform into core/shell $\mathrm{Co}_{\mathrm{hcp}} / \mathrm{CoO}$ nanocrystals.

It is noticeable that the superlattice keeps its integrity as no coalescence is observed between the "new" building blocks. The same exposure to air made on the same population of hcp-Co single crystals but that are in a disordered and/or isolated fashion gives rise to fully oxidized $\mathrm{CoO}$ nanocrystals that tend to coalesce (Fig. $5 \mathrm{~F}$ and inset).

This study clearly evidences the enhanced stability against oxidation of hcp-Co single crystals due to 2D self-organization. The robustness of Co nanocrystals observed in the $2 \mathrm{D}$ organizations is attributed to a decrease in the permeability of the dodecanoic acid chains to $\mathrm{O}_{2}$ molecules. From a magnetic point of view, the core/shell $\mathrm{Co}_{\text {hcp }} / \mathrm{CoO}$ nanocrystals are highly desirable as we can expect an additional exchange anisotropy compared to the hcp-Co nanocrystals.

\section{3D superlattices of Co nanocrystals. Physical properties}

\subsection{Evidence of super-spin glass behavior in $3 D$ supracrystals of Co nanocrystals}

In a conventional atomic spin glass, the lack of long range magnetic order arising from magnetic frustration or highly dilute magnetic ions, leads to interesting behaviour such as aging, rejuvenation and memory effects $[47,48]$. In a magnetic nanoparticle system, if the nanoparticles are small enough to have a single magnetic domain, each nanoparticle will act like a giant or "super" spin. In sufficiently concentrated systems, these "superspins" can interact via long range dipolar interactions, whose random nature leads to a highly disordered and frustrated magnetic state analogous to that in atomic spin glasses. Until now, the interacting systems that have been investigated are disordered systems, where the nanocrystals are not in a regular array [49-53]. Therefore, the question that arises is what is the influence of the long-range ordering of Co nanocrystals in a $3 \mathrm{D}$ fcc array on the superspin glass behavior.

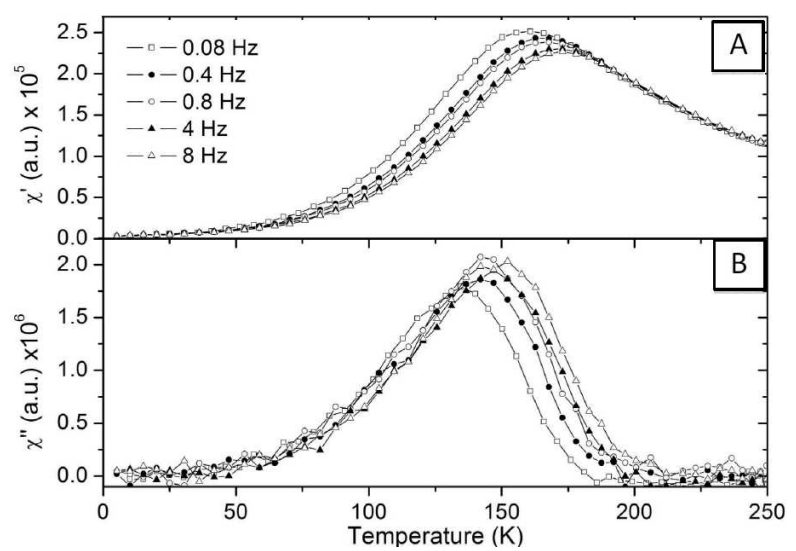

Fig. 6. (A) In phase and (B) out of phase part of the ac susceptibility versus temperature, measured at frequencies between 0.08 and $8 \mathrm{~Hz}$ for $8 \mathrm{~nm}$ Co polycrystals.

Fcc supracrystals were prepared by slowly evaporating a colloidal solution of $8 \mathrm{~nm}$ Co polycrystals on highly oriented pyrolytic graphite as presented in Sect. 2.2 (Fig. 1C,D) [34]. The ZFC magnetisation increases with increasing temperature up to a maximum, $T_{\mathrm{B}}$, above which the behaviour is paramagnetic [47]. In this case, $T_{\mathrm{B}}(130 \mathrm{~K})$ is significantly larger than that observed for a dilute system of Co nanocrystals of the similar size [54] due to the strong magnetic dipolar interactions between the nanocrystals in the supracrystal. The FC magnetisation curve is nearly temperature independent below $T_{\mathrm{B}}$, characteristic of spin glass behaviour [47].

In less interacting systems where the behaviour is superparamagnetic the FC magnetisation curve is not temperature-independent below $T_{\mathrm{B}}$ but increases with decreasing temperature [55].

Figure 6 A,B shows the in-phase $\left(\chi^{\prime}\right)$ and out-of-phase $\left(\chi^{\prime \prime}\right)$ components of the ac susceptibility versus temperature respectively, measured in a range of ac frequencies varying by 2 decades in magnitude. The $\chi^{\prime}$ susceptibility shows a clear frequency dependence, where the temperature at which the maximum susceptibility is observed $\left(T_{\text {peak }}\right)$ increases with increasing frequency. The $\chi^{\prime \prime}$ susceptibility also shows frequency dependence, where the maximum slope for a given frequency corresponds to $T_{\text {peak }}$ in the $\chi^{\prime}$ curve.

Frequency dependence in an ac measurement is observed both in superparamagnetic and superspin glass materials. In order to differentiate between these two types of behaviour, one can analyse quantitatively the change in $T_{\text {peak }}$ with frequency.

For a superparamagnet, where dipolar interactions between the magnetic moments are negligible, the fre- 
quency dependence should follow an Arrhenius law $\tau=$ $\tau_{0} \exp \left(E_{\mathrm{a}} / k_{\mathrm{B}} T\right)$ where $E_{\mathrm{a}}$ is the anisotropy energy, $\tau$ is the inverse of the measurement frequency and $\tau_{0}$ is an attempt time. By plotting $\log _{10} \tau$ versus $1 / T_{\text {peak }}$ and fitting the data to a straight line, the value of $\tau_{0}$ can be extracted and in this case we find $\tau_{0}=10^{-31} \mathrm{~s}$. This unphysically small value indicates that this system is not best described by simple energy barrier blocking and thermal activation and a different approach is needed. Hence, we have tried fitting the data to a critical power law $\tau=\tau^{*}\left(T_{\text {peak }} / T_{\mathrm{g}}-1\right)^{-z \nu}$, where $T_{\mathrm{g}}$ was taken as the maximum in the dc ZFC magnetization curve. Fitting the data yielded $\tau^{*}=10^{-9 \pm 3} \mathrm{~s}$ and $z \nu=12 \pm 2$. This value of $\tau *$ is in good agreement with values found for spin glasses, and $z \nu$, although slightly high, is also compatible within error to that expected for spin glasses [48, 55, 56].

Spin glass and superspin glass materials are known to show aging and memory effects, which can be demonstrated by a simple dc magnetization experiment. The sample is zero field cooled from above $T_{\mathrm{g}}$ to a temperature $T_{\mathrm{s}}$ typically equal to $0.7 T_{\mathrm{g}}$ where a waiting time of $t_{\mathrm{w}}=10^{4} \mathrm{~s}$ is imposed before continuing cooling down to low temperature. A small field is then applied and the magnetization is measured on heating. A deviation from the reference $\mathrm{ZFC}$ curve (with no stop during cooling) is observed at $T_{\mathrm{s}}$, which is known as a "memory dip", so called as the system has "remembered" the relaxation towards a zero magnetization value (aging) that occurred during the cooling process.

The results of these ac and dc susceptibility investigations provide strong evidence for superspin glass behaviour in these fcc Co nanocrystal supracrystals.

\subsection{Magnetic intrinsic property of 3D superlattices of Co nanocrystals}

Owing to the ability of controlling the mesoscopic ordering of 3D assemblies of Co nanocrystals (see Sect. 2.4), we can carry out a carefully controlled comparative investigation of the magnetic properties of long-range fcc supracrystals and disordered assemblies, both composed of the same population of nanocrystals (the same size and the same size distribution).

Co nanocrystals used for this study are characterized by a mean size and size distribution of $7.5 \mathrm{~nm}$ and $9.4 \%$, respectively. Figure $7 \mathrm{~A}$ shows the $\mathrm{FC}$ and $\mathrm{ZFC}$ magnetization versus temperature curves, normalized to the blocking temperature, measured with an applied field of 20 Oe, of fcc supracrystals (black) and disordered assemblies (red). Whatever the mesoscopic ordering is, $T_{\mathrm{B}}$ does not change significantly, its value is found around $100 \mathrm{~K}$. However, as it can be observed in Fig. 7A, the ZFC peak is significantly narrower for the supracrystals. The width of the ZFC peak is dependent on the distribution of energy barriers, $E_{\mathrm{b}}$, in the system: a larger distribution gives a broader peak. The barrier energy is the sum of the anisotropy energy $\left(E_{\mathrm{a}}=k_{\mathrm{a}} V\right.$ where $k_{\mathrm{a}}$ is the anisotropy constant and $V$ is the nanocrystal volume) and the interparticle dipole interaction energy $\left(E_{\mathrm{dd}}\right)[57]$.
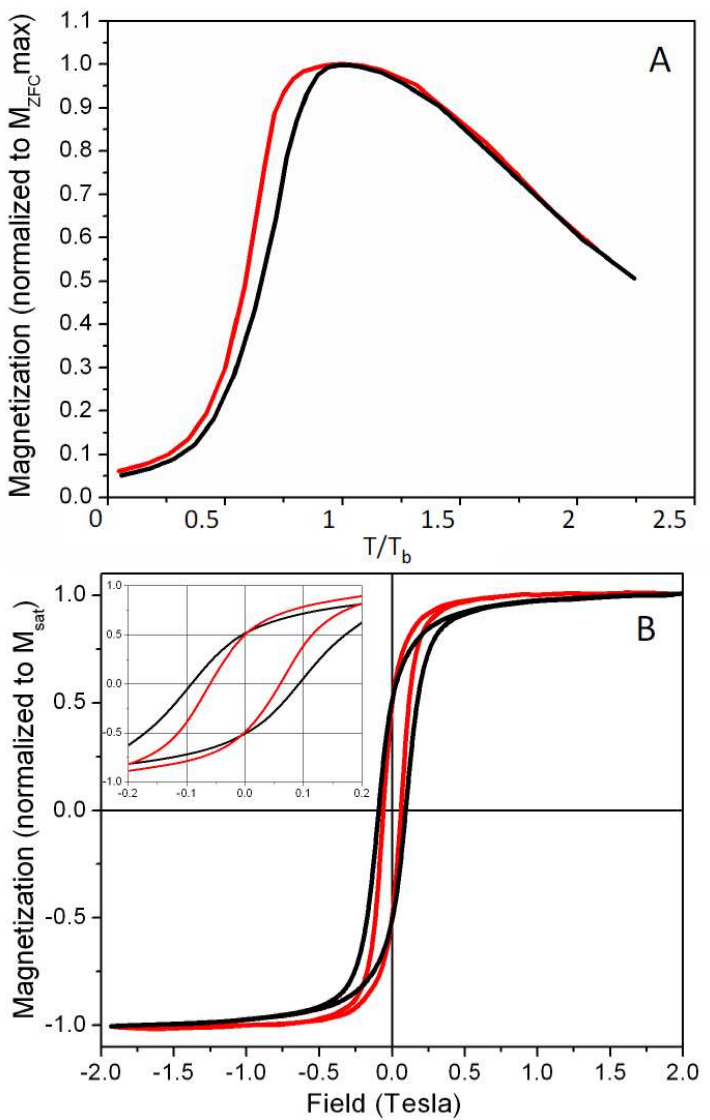

Fig. 7. (A) ZFC $M$ vs. $T / T_{\mathrm{B}}$ curves of fcc supracrystals (black) and a disordered $3 \mathrm{D}$ assembly at $T=5 \mathrm{~K}$ (red). (B) $M$ vs. $H$ curves normalized to $M_{\mathrm{s}}$ of fcc supracrystals (black) and disordered 3D assembly (red). Inset: magnification of the low field region.

As the nanocrystals used to form the two samples come from the same synthesis batch, we can discount any effects of nanocrystal volume distribution and anisotropy. We therefore explain the difference in the distribution of $E_{\mathrm{b}}$ by the change in the mesoscopic ordering in the assemblies. As pointed out in Ref. [58], dipolar forces have a strong directional dependence and, consequently, dipolar interactions in the assembly should be sensitive to the detailed geometrical arrangement of the nanocrystals.

In the supracrystals, Co nanocrystals are long-range ordered in an fcc structure, i.e., their environment is highly uniform related to the coordination and interparticle gap. The disordered assembly has no long-range order and consists of very small crystallized domains likely separated by amorphous material. In this case, the nanocrystals have a much less uniform environment than the supracrystals, this leads to a greater distribution of $E_{\mathrm{dd}}$ (and hence $E_{\mathrm{b}}$ ) compared to the supracrystals. We acknowledge that this effect of order is fairly subtle, however, we have found that it is highly reproducible.

Figure 7B shows the magnetization versus field curves for the supracrystal (black) and disordered (red) samples 
at $5 \mathrm{~K}$. In both cases, saturation is reached at around $1 \mathrm{~T}$ and hysteresis is observed. For the ordered sample, we find that the coercivity, $H_{\mathrm{c}}$ is larger than for the disordered sample (900 against $600 \pm 50 \mathrm{Oe}$ ) and that the latter saturates at slightly lower fields. This faster approach to saturation seen for the disordered sample is coherent with a $\Delta M \approx H^{-1 / 2}$ behavior as observed in amorphous ferromagnets [59].

Experimental studies on these materials and on analogous nanoparticle films have shown that a higher anisotropy leads to a slower approach to saturation as we observe for the supracrystal sample [60, 61]. We therefore propose that the supracrystals have a higher sample anisotropy compared to the disordered sample (as is also confirmed by the higher $H_{\mathrm{c}}$ ) due to the increased mesoscopic order. This behavior presents an analogy with bulk crystalline materials where higher atomic crystallographic order leads to an increase in anisotropy.

This result constitutes the first intrinsic magnetic property due to the mesoscopic order in 3D assemblies of metallic nanocrystals.

\section{Conclusion}

In this paper, we show that we can control both the mesoscopic ordering of fcc supracrystals composed of Co nanocrystals and the Co crystallinity. Hence, remarkable intrinsic collective magnetic properties have been discovered. We give clear evidence of the enhanced stability against oxidation due to $2 \mathrm{D}$ self-organization of hcp-Co single crystals. When the nanocrystals are ordered in a close-packed array, the formation of core/shell $\mathrm{Co}_{\mathrm{hcp}} /$ $\mathrm{CoO}$ nanocrystals occurs whereas nanocrystals in disordered areas or isolated on the substrate tend to fully oxidize to pure $\mathrm{CoO}$. We show that annealing the $2 \mathrm{D}$ and $3 \mathrm{D}$ superlattices of Co nanocrystals leads to an increase in the nanocrystal anisotropy with no damage to the superlattices making these artificial solids almost ferromagnetic at room temperature. Finally, we show evidence for super-spin glass behavior in fcc supracrystals of Co polycrystals.

\section{Acknowledgments}

Special thanks are due to Profs. M.P. Pileni and G. Van Tendeloo, and Drs. P.A. Albouy, D. Parker, C. Salzemann, and M. Walls.

\section{References}

[1] M.P. Pileni, J. Phys. Chem. B 105, 3358 (2001).

[2] S. Srivastava, N.A. Kotov, Soft Matter 5, 1146 (2009).

[3] K.J.M. Bishop, C.E. Wilmer, S. Soh, B.A. Grzybowski, Small 5, 1600 (2009).

[4] E.V. Shevchenko, D.V. Talapin, N.A. Kotov, S. O'Brien, C.B. Murray, Nature 439, 55 (2006).

[5] M.A. Correa-Duarte, J. Perez-Juste, A. Sanchez-Iglesias, M. Giersig, L.M. Liz-Marzan, Angew. Chem. Int. Ed. 44, 4375 (2005).
[6] Q.L. Zhang, S. Gupta, T. Emrick, T.P. Russell, J. Am. Chem. Soc. 128, 3898 (2006).

[7] F.A. Aldaye, A.L. Palmer, H.F. Sleiman, Science 321, 1795 (2008).

[8] A. Tao, J.X. Huang, P. Yang, Acc. Chem. Res. 41, 1662 (2008).

[9] V. Germain, M.P. Pileni, Adv. Mater. 17, 1424 (2005).

[10] A. Taleb, F. Silly, A.O. Gusev, F. Charra, M.P. Pileni, Adv. Mater. 12, 633 (2000).

[11] V. Russier, C. Petit, J. Legrand, M.P. Pileni, Phys. Rev. B 62, 3910 (2000).

[12] V. Russier, C. Petit, M.P. Pileni, J. Appl. Phys. 93, 10001 (2003).

[13] C. Petit, J. Legrand, V. Russier, M.P. Pileni, J. Appl. Phys. 91, 1502 (2002).

[14] I. Lisiecki, D. Parker, C. Salzeman, M.P. Pileni, Chem. Mater. 19, 4030 (2007).

[15] A. Taleb, C. Petit, M.P. Pileni, J. Phys. Chem. B 102, 2214 (1998).

[16] P. Yang, H. Portalès, M.P. Pileni, Phys. Rev. B 81, 215405 (2010).

[17] N. Zaitseva, Z.R. Dai, F.R. Leon, D. Krol, J. Am. Chem. Soc. 12, 10221 (2005).

[18] A. Courty, A. Mermet, P.A. Albouy, E. Duval, M.P. Pileni, Nat. Mater. 4, 395 (2005).

[19] E. Duval, A. Mermet, A. Courty, P.A. Albouy, M.P. Pileni, Phys. Rev. B 72, 085439-1/4 (2005).

[20] I. Lisiecki, V. Halte, C. Petit, M.P. Pileni, J.Y. Bigot, Adv. Mater. 20, 4176 (2008).

[21] J.V. Sanders, Nature 204, 1151 (1964).

[22] I. Lisiecki, M. Walls, D. Parker, M.P. Pileni, Langmuir 24, 4295 (2008).

[23] I. Lisiecki, S. Turner, S. Bals, M.P. Pileni, G. Van Tendeloo, Chem. Mater. 21, 2335 (2009).

[24] E. Klecha, D. Ingert, M.P. Pileni, Langmuir 25, 2824 (2009).

[25] E. Klecha, D. Ingert, M.P. Pileni, J. Phys. Chem. Lett. 1, 1616 (2010).

[26] B.D. Terris, T. Thomson, J. Phys. D, Appl. Phys. 38, R199 (2005).

[27] Acc. Chem. Res., special issue on Nanoscale Materials 32 (1999).

[28] D.N. Lambeth, E.M.T. Velu, G.H. Bellesis, L.L. Lee, D.E. Laughlin, J. Appl. Phys. 79, 4496 (1996).

[29] I. Lisiecki, M.P. Pileni, Langmuir 19, 9486 (2003).

[30] C. Petit, P. Lixon, M.P. Pileni, Langmuir 7, 2620 (1991).

[31] H.T. Yang, C.M. Shen, Y.K. Su, T.Z. Yang, H.J. Gao, Nanotechnology 15, 70 (2004).

[32] G.N. Glave, K.J. Klabunde, C.M. Sorensen, G.C. Hadjipanayis, Langmuir 9, 162 (1993).

[33] J. Aizenberg, G. Lambert, S. Weiner, L. Addadi, J. Am. Chem. Soc. 124, 32 (2002).

[34] I. Lisiecki, P.A. Albouy, M.P. Pileni, Adv. Mater. 15, 712 (2003).

[35] I. Lisiecki, C. Salzemann, D. Parker, P.A. Albouy, M.P. Pileni, J. Phys. Chem. C 111, 12625 (2007). 
[36] D. Weller, A. Moser, IEEE Trans. Magn. 35, 4423 (1999).

[37] D.A. Thompson, J.S. Best, IBM J. Res. Dev. 44, 311 (2000).

[38] V. Skumryev, S. Stoyanov, Y. Zhang, G. Hadjipanayis, D. Givord, J. Nogués, Nature 423, 850 (2003).

[39] D.D. Schwalom, S. von Molnar, in: Nanotechnology, Ed. G.L. Timp, Springer, New York 1999, Ch. 12, p. 437.

[40] S. Sun, C.B. Murray, J. Appl. Phys. 85, 4325 (1999).

[41] H. Zeng, S. Sun, R.L. Sandstrom, C.B. Murray, J. Magn. Magn. Mater. 266, 227 (2003).

[42] A.C.C. Yu, M. Mizuno, Y. Sasaki, H. Kondo, K. Hiraga, Appl. Phys. Lett. 81, 3768 (2002).

[43] H.T. Yang, C.M. Shen, Y.K, Su, T.Z, Yang, H.J. Gao, Y.G. Wang, Appl. Phys. Lett. 82, 4729 (2003).

[44] X. Nie, J.C. Jiang, E.I. Meletis, L.D. Tung, L. Spinu, J. Appl. Phys. 93, 4750 (2003).

[45] D. Parker, I. Lisiecki, C. Salzemann, M.P. Pileni, J. Phys. Chem. C 111, 12632 (2007).

[46] I. Lisiecki, P.A. Albouy, M.P. Pileni, J. Phys. Chem. B 108, 20050 (2004).

[47] D. Parker, I. Lisiecki, M.P. Pileni, J. Phys. Chem. Lett. 1, 1139 (2010).

[48] J.A. Mydosch, Spin Glasses: An Experimental Introduction, Taylor \& Francis, London 1993.

[49] C. Djurberg, P. Svedlindh, P. Nordblad, M.F. Hansen, F. Bodker, S. Morup, Phys. Rev. Lett. 79, 5154 (1997).
[50] H. Mamiya, I. Nakatani, T. Furubayashi, Phys. Rev. Lett. 80, 177 (1998).

[51] T. Jonsson, P. Svedlindh, M.F. Hansen, Phys. Rev. Lett. 81, 3976 (1998).

[52] S. Sahoo, O. Petracic, Ch. Binek, W. Kleemann, J.B. Sousa, S. Cardoso, P.P. Freitas, Phys. Rev. B 65, 134406 (2002).

[53] D. Parker, V. Dupuis, F. Ladieu, J.P. Bouchaud, E. Dubois, R. Perzynski, E. Vincent, Phys. Rev. B 77, 104428 (2008)

[54] C. Petit, Z.L. Wang, M.P. Pileni, J. Phys. Chem. B 109, 15309 (2005).

[55] J.L. Dormann, Phys. Rev. B 53, 14291 (1996).

[56] M.F. Hansen, P.E. Jonsson, P. Nordblad, P. Svedlindh, J. Phys. Condens. Matter. 14, 4901 (2002)

[57] J.L. Dormann, L. Spinu, E. Tronc, J.P. Julivet, F. Lucari, F. D'orazio, D. Fiorani, J. Magn. Magn. Mater. 183, L255 (1998).

[58] D. Kechrakos, K.N. Trohidou, Phys. Rev. B 58, 12169 (1998).

[59] E.M. Chudnovsky, J. Appl. Phys. 64, 5770 (1988).

[60] J. Filippi, V.S. Amaral, B. Barbara, Phys. Rev. B 44, 284 (1991).

[61] L. Thomas, J. Tuaillon, J.P. Perez, V. Dupuis, A. Perez, B. Barbara, J. Magn. Magn. Mater. 437, 140 (1995). 